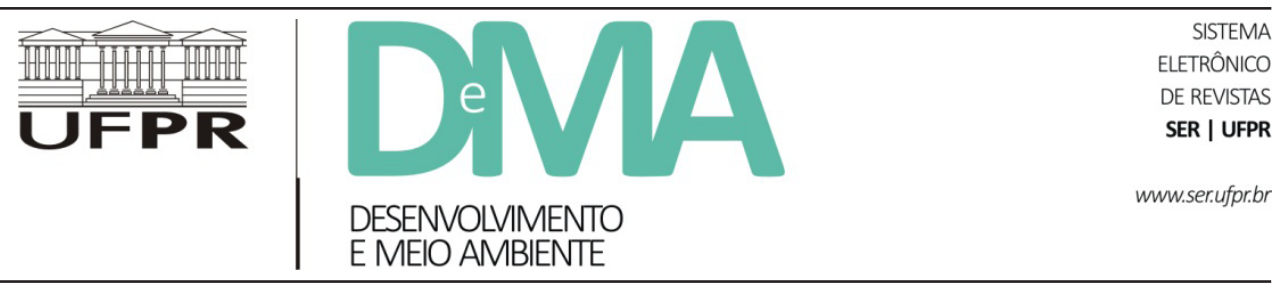

\title{
Estudo etnofarmacológico sobre Lafoensia replicata Pohl. no leste do Maranhão, Brasil: uma promissora espécie para bioprospecção
}

\section{Ethno-pharmacological Study about Lafoensia replicata Pohl. in East Maranhão, Brazil: A Promising Species for Bioprospection}

\author{
Francisco Chagas Bila SOBRINHO ${ }^{1}$, Alyson Luiz Santos ALMEIDA ${ }^{1}$, Julio Marcelino MONTEIRO ${ }^{*}$ \\ ${ }^{1}$ Universidade Federal do Piaú (UFPI) - Campus Amílcar Ferreira Sobral, Floriano, PI, Brasil. \\ *E-mail de contato: juliommonteiro@yahoo.com.br
}

Artigo recebido em 11 de maio de 2016, versão final aceita em 18 de outubro de 2016.

RESUMO: Esta pesquisa objetivou realizar estudo sobre os usos terapêuticos da mangabeira (Lafoensia replicata Pohl.), uma importante planta do cerrado nordestino. Foram realizadas 32 entrevistas com informantes da comunidade Manga, Barão de Grajaú, interior do Maranhão. Os entrevistados, 18 mulheres e 14 homens, informaram oito usos diferentes para a planta. Quanto ao número de indicações de uso, não houve diferenças significativas entre os gêneros: oito indicações descritas pelas mulheres e sete pelos homens. Com relação a distinções no conhecimento entre as classes de idade propostas para análise, também não houve distinções estatísticas. A parte mais indicada como usada foram as cascas do caule. Todos os informantes indicaram consensualmente que o remédio preparado a partir da planta deve ser administrado por via oral, e mais da metade dos informantes mencionou que a planta não apresenta restrições ao consumo (53\%). Contudo, 17 entrevistados afirmaram que o tratamento é contraindicado durante a gravidez e, ainda, cinco pessoas não recomendaram o uso para crianças. Diante de tudo isso, não foi possível, com as informações etnofarmacológicas deste estudo, alicerçar a literatura especializada, já que há insuficiência de pesquisas com a espécie estudada. Há alguns estudos enfocando usos e atividades de L. pacari A. St.-Hil. Dessa forma, esse estudo recomenda também L. replicata Pohl. como uma potencial espécie terapêutica.

Palavras-chave: mangabeira; medicina tradicional; etnobotânica.

ABSTRACT: This work aimed at carrying out a study on the local therapeutic uses of mangabeira (Lafoensia replicata Pohl.), an important plant in the northeastern cerrado. 32 interviews were conducted with informants from the community Manga, Barão do Grajaú, Maranhão. Respondents, 18 women and 14 men, reported eight different uses for the plant. Regarding the number of instructions for use, there were no significant differences between genders, eight nominations described by women and seven by men. Regarding the distinctions of knowledge among the age group proposed for the analysis, there were no statistical differences. The plant part 
most indicated for use was the stem bark. All informants indicated consensually that the medicine prepared from the plant should be administered orally and over half of the respondents mentioned that the plant had no restrictions on consumption (53\%). However, 17 respondents said that the treatment is contraindicated during pregnancy, and even five people did not recommend the use on children. It was not possible to substantiate the ethno-pharmacological information in this study with the literature since there is insufficient research on this species. There is some research focusing on uses and activities of Lafoensia pacari A. St.-Hil, therefore, this study also recommends $L$. replicata Pohl. as a potential therapeutic species.

Keywords: mangabeira; traditional medicine; ethno-botany.

\section{Introdução}

O homem, desde os primórdios, vem utilizando recursos naturais para aliviar ou curar diversas doenças (Albuquerque, 1997) e ainda hoje muitos utilizam plantas como atividade terapêutica por todo o mundo (WHO, 2014). De modo geral, populações que residem em áreas afastadas dos centros urbanos, próximas a matas ou reservas florestais, acumularam amplo conhecimento sobre terapias a partir da vegetação disponível e os etnobotânicos procuram tais informações sobre as relações homem-planta. Assim, interessante ferramenta para o manejo desses recursos pode ser confeccionada e, sobre isso, Christo et al. (2006) afirmaram que uma das ações imprescindíveis para a realização de programas de conservação da biodiversidade é a inclusão de comunidades tradicionais no processo de proteção e manejo sustentável.

Algumas espécies do semiárido nordestino são popularmente importantes pelas significativas concentrações de compostos bioativos em determinadas partes (Agra et al., 2007; Araujo et al., 2008; Melo et al., 2011a; 2001b; Monteiro et al., 2011; Almeida et al., 2012; Soldati \& Albuquerque, 2012; Monteiro et al., 2014). Há grupos de espécies nativas do semiárido com bom número de estudo, gozando da atenção dos pesquisadores, como Myracrodruon urundeuva Allemão, Anadenanthera colubrina (Vell.) Brenan, Stryphnodendron rotundifolium Mart., por exemplo (Ramos et al., 2012; Monteiro et al., 2006; 2014; Oliveira et al., 2014; Barros et al., 2016). Porém, outras plantas conhecidas e utilizadas por sertanejos, na terapia popular, ainda têm pouca atenção dos cientistas, como a mangabeira (Lafoensia replicata Pohl.), objeto do presente estudo. Na verdade, há estudos sobre o uso e a atividade biológica da espécie $L$. pacari A. St.-Hil. (Solon et al., 2000; Galdino et al., 2009; Guimarães et al., 2010; Porfírio et al., 2009). A atividade antibacteriana do extrato de $L$. pacari A. St.-Hil. foi verificada e constatou-se que $96,4 \%$ das linhagens utilizadas foram suscetíveis ao extrato da folha da planta (Porfírio et al., 2009). Galdino et al. (2009) verificaram atividade antidepressiva em ratos do extrato etanólico das cascas do caule de L. pacari A. St.-Hil. Os autores relataram que a depressão é um dos males mais expressivos da atualidade, de acordo com a Associação Brasileira de Psiquiatria, e, assim, a busca de novos compostos se faz necessária. Num estudo orientado para plantas do cerrado, Lima et al. (2015) testaram atividade contra as formas promastigotas de Leishmania amazonensis e verificaram que o extrato hidroalcoólico L. pacari A. St.-Hil apresentou atividade leishmanicida significativa, confirmando sua eficácia. Essa gama de atividades supracitadas para L. pacari A. St.-Hil. pode ser um indicativo de importância para L. replicata Pohl. Assim, esse estudo é interessante por resgatar o conhecimento de uma importante 
espécie muito usada por comunidades tradicionais e propor informações para futuros projetos envolvendo a coleta de plantas medicinais associada a manejo sustentado para a área.

Diante disso, esta pesquisa objetivou realizar um estudo sobre os usos terapêuticos da mangabeira (Lafoensia replicata Pohl.), a parte usada da planta, a forma de administração, o modo de preparo e as restrições quanto ao uso do remédio preparado numa comunidade no interior do estado do Maranhão.

\section{Metodologia}

\section{1. Área de estudo}

O município de Barão de Grajaú, MA, localizado na mesorregião leste maranhense, microrregião das chapadas do alto Itapecuru, na margem esquerda do rio Parnaíba, com altitude da sede, em relação ao nível do mar, de 108 metros, apresenta clima tropical ameno. As temperaturas ficam entre 27 e 37 graus centígrados e as chuvas são bem definidas no verão e raras no inverno (IBGE, 2014). Elas se concentram entre os meses de novembro e abril, e a temperatura aumenta entre os meses de maio e outubro. O cerrado é a vegetação predominante na região (IBGE, 2014). Na agricultura, os destaques são arroz, coco-babaçu, buriti e juçara. A cidade exporta óleos de amêndoas e babaçu, algodão em pluma e arroz (IBGE, 2014).

A área de estudo deste trabalho é a comunidade Manga, que se localizada a $45 \mathrm{~km}$ do município de Barão de Grajaú. De acordo com os moradores mais antigos, o nome Manga foi dado por causa da localização no trecho mais estreito do rio Parnaíba, utilizado para transportar o gado entre suas margens. A comunidade conta com uma equipe do Programa Saúde Familiar (PSF), que se encontra presente durante toda a semana na comunidade. Quando ocorrem casos graves de saúde, os moradores são deslocados para o município de Barão de Grajaú. De acordo com essa equipe, há 40 famílias fixas e cerca de 30 que visitam a comunidade no período da "prainha" e de festejos. Os moradores são de origem rural e em sua maioria sobrevivem da agricultura ou da pesca. $\mathrm{O}$ acesso à comunidade estudada se dá por via fluvial, através do rio Parnaíba, e por via terrestre, sendo parte pela BR-230 até o km 35 e parte por estrada não asfaltada, totalizando os $17 \mathrm{~km}$ restantes até a comunidade.

\subsection{Coleta e análise dos dados}

Inicialmente, estabeleceu-se uma relação amistosa com a população-alvo, por meio de uma explanação detalhada de todos os objetivos da pesquisa. Após conhecerem os objetivos, todos os informantes foram convidados a assinar um termo de consentimento livre e esclarecido, cedendo a permissão para a realização da pesquisa de acordo com a resolução vigente. $O$ presente trabalho apresenta o Certificado de Apresentação para Apreciação Ética (CAAE 46264515.0.0000.5214), de acordo com o Comitê de Ética em Pesquisa com Seres Humanos da UFPI.

Em seguida, foram realizadas entrevistas com informantes na área estudada, em suas próprias residências, empregando-se a técnica da entrevista semiestruturada, em que o pesquisador elabora perguntas que servem como um roteiro que abrange uma lista de tópicos, permitindo aprofundar elementos que possam surgir durante a entrevista (Albuquerque et al., 2014). A coleta de informações sobre a planta se deu entre fevereiro e setembro de 2014. A "viagem de campo dividida" (Titiev, 2000) permitiu acrescentar informações de distintas 
épocas do ano, bem como suplantar as lacunas das viagens anteriores, como, por exemplo, entrevistas com informantes que não se encontravam presentes no momento da visita.

Os informantes foram questionados sobre os usos terapêuticos de L. replicata Pohl., conhecida localmente como mangabeira. Também se pesquisou sobre a parte usada da planta, a forma de administração, o modo de preparo e as restrições ao seu uso. As entrevistas se basearam em formulários que em sua primeira parte continham perguntas sobre informações pessoais dos entrevistados, e a parte seguinte possuía questões específicas sobre a espécie (Albuquerque et al., 2014). O material botânico foi, com o auxílio de alguns informantes, coletado, herborizado, identificado e depositado no Laboratório de Botânica do Campus Amílcar F. Sobral, UFPI.

Além disso, ferramentas quantitativas foram utilizadas como medidas de conhecimento para avaliar o consenso entre as pessoas em relação aos usos (Byg \& Baslev, 2001; Oliveira et al., 2014). Para analisar o conhecimento local dos informantes sobre a espécie, foram utilizados os índices: valor de consenso para diversidade do uso (VCDU) e valor de consenso para parte da planta (VCPP) (Byg \& Baslev, 2001; Oliveira et al., 2014). Diferenças significativas foram verificadas em função dos índices propostos, do gênero e das classes de idade, com base no Teste de Kruskal-Wallis a 5\%, e processadas pelo programa BioEstat 5.0 (Ayres et al., 2007). Para as análises, os informantes foram agrupados em três classes de idade: menores de 42 anos, entre 42 e 62 anos e maiores de 62. Para o valor de diversidade de uso (VDU), os tipos de usos terapêuticos, conforme forem citados pelos informantes, serão enquadrados nas seguintes categorias: desordens gastrointestinais, dermatológicas, urinárias, infecciosas ou inflamatórias e respiratórias (Oliveira et al., 2014). Também foram utilizadas porcentagens para analisar os dados etnobotânicos reportados para a planta estudada.

\section{Resultados e discussão}

\subsection{Informações socioculturais dos entrevistados na comunidade Manga, Barão de Grajaú, MA}

Foram visitadas 40 residências. Oito não manifestaram interesse em participar ou não conheciam a planta. Um total de 32 pessoas conheceu e indicou Lafoensia replicata Pohl. (mangabeira) para uso fisioterápico. As características dos informantes estão listadas na Tabela 1. Foram levadas em consideração as seguintes classes de idade: cinco informantes $(15,6 \%)$ tinham menos de 42 anos de idade no momento da visita, $20(62,5 \%)$ estavam entre 42 e 62 anos de idade e sete $(21,8 \%)$, acima de 62 anos de idade. Com relação à ocupação, 27 (84\%), praticamente a maioria das pessoas, estão envolvidos na agricultura, dois $(6,2 \%)$ são pescadores, duas $(6,2 \%)$ são professoras e um $(3,1 \%)$ é mecânico. A maioria dos informantes apresentou ensino fundamental incompleto (59,3\%), nove deles são analfabetos e as duas professoras têm curso superior completo (Tabela 1).

Os entrevistados, 18 mulheres (56,27\%) e 14 homens $(43,75 \%)$, informaram oito usos diferentes para a planta. Quanto ao número de indicações de uso, não houve diferenças significativas entre os gêneros: oito indicações descritas pelas mulheres e sete pelos homens $(H=1,77 ; p=0,18)$. Oliveira et al. (2014) também não encontraram distinções significativas entre os gêneros, num estudo etnofarmacológico sobre o Stryphnodendrum rotundifolium Mart., na região do Araripe, Nordeste do Brasil. 
TABELA 1 - Número de informantes, idade, ocupação, nível de instrução e gênero dos entrevistados na comunidade Manga, Barão de Grajaú, MA

\begin{tabular}{lcc}
\hline & $\begin{array}{c}\text { Número de } \\
\text { entrevistados } \\
(\mathbf{n}=\mathbf{3 2})\end{array}$ & $\mathbf{\%}$ \\
\hline Idade & 5 & 15,6 \\
$\quad$ Abaixo de 42 & 20 & 62,5 \\
$\quad$ Entre 42 e 62 & 7 & 21,8 \\
$\quad$ Acima de 62 & & \\
Ocupação & 27 & 84,3 \\
$\quad$ Agricultores & 2 & 6,2 \\
Pescadores & 3 & 9,3 \\
$\quad$ Outros & & \\
Nível de instrução & 9 & 28,1 \\
$\quad$ Analfabeto & 19 & 59,3 \\
$\quad$ Ensino fundamental incompleto & 1 & 3,1 \\
$\quad$ Ensino fundamental completo & 1 & 3,1 \\
$\quad$ Ensino médio & 2 & 6,2 \\
$\quad$ Ensino superior & & \\
Gênero & 14 & 43,7 \\
$\quad$ Masculino & 18 & 56,2 \\
$\quad$ Feminino & & \\
\hline
\end{tabular}

Pouco mais da metade dos entrevistados é do sexo feminino no presente estudo (Tabela 1). De acordo com alguns autores, há distinções no conhecimento entre gêneros, cabendo, principalmente, às mulheres o diagnóstico e o tratamento de doenças em certas comunidades pesquisadas (Voeks \& Nyawa, 2001; Voeks \& Leony, 2004; Torres-Avilez et al., 2014). Para os homens, há o direcionamento de trabalhos externos à residência (Lucena et al., 2012) e que também implicam o uso da força física, como o uso madeireiro na construção, por exemplo (Torres-Avilez et al., 2014). Segundo Voeks (2007), as mulheres destacaram-se quanto ao uso de plantas terapêuticas e isso pode ser decorrente de vivências com manejo dos quintais e tratamento, via fitoterapia, de familiares e pessoas mais próximas (Silva et al., 2011; Voeks, 2007). Em contrapartida, outras pesquisas não encontraram tais distinções entre homens e mulheres (Monteiro et al., 2006; Giraldi \& Hanazaki, 2010; Baptistel et al., 2014). Giraldi \& Hanazaki (2010), no Sertão do Ribeirão, SC, constataram que o gênero não influenciou a pesquisa, porém esse resultado pode ser explicado devido ao baixo número de entrevistas realizadas, destacaram as autoras. Baptistel et al. (2014) entrevistaram 32 pessoas em estudo etnobotânico no sul do Piauí e, concernente ao conhecimento sobre plantas medicinais, também não encontraram distinções entre gêneros. Já Monteiro et al. (2006), em pesquisa realizada com 101 informantes acerca de duas espécies medicinais numa comunidade do interior de Pernambuco, também não perceberam distinções significativas quanto ao gênero. Assim, sabe-se que uma amostra consistente é condição imprescindível para um bom estudo. No entanto, outros fatores podem influenciar as distinções entre características socioculturais, como idade, tempo de moradia ou escolaridade.

Outro aspecto interessante é que o conhecimento sobre a espécie estudada está homogeneamente distribuído entre os entrevistados deste estudo. Não foi notado um especialista ou grande conhecedor que se destacasse dos demais. Sobre isso, Begossi et al. (2002) afirmaram que a perda de um grande conhecedor de plantas medicinais pode afetar os sistemas locais de tratamento, quando estes existem em comunidades pequenas, principalmente se esse saber não foi repassado.

Com relação a distinções no conhecimento entre as classes de idade propostas para análise, também não houve distinções estatísticas (mulheres com menos de 42 anos; entre 42 e 62 anos; e acima de 62 anos $-\mathrm{H}=0,45 ; \mathrm{p}=0,79$ - homens entre 42 e 62 anos e acima de 62 anos $-\mathrm{H}=0,0 ; \mathrm{p}$ $=0,94)$. Não houve homens entrevistados abaixo de 42 anos de idade. É amplamente aceitável que as pessoas mais idosas acumulem maior quantidade 
de informações sobre o ambiente em que vivem em comparação com as mais novas. Contudo, não se perceberam neste trabalho distinções entre as classes de idades propostas. Sendo assim, estudos futuros são necessários para verificar quais fatores interferem no conhecimento acumulado, como número de informantes, gênero, idade ou grau de escolaridade.

\subsection{Diversidade de uso e consenso para a parte da planta de L. replicata Pohl. na comunidade Manga, MA}

Com relação aos usos de $L$. replicata Pohl., foi calculado o VCDU para todas as indicações (Tabela 2). Os entrevistados citaram, em maior quantidade, problemas relacionados ao fígado, seguidos de problemas de inflamação em geral, uso como cicatrizante, cura ou alívio para os problemas dos rins. Logo em seguida, gastrite, pressão alta, dor de cabeça e dor de barriga.

TABELA 2 - Valor de consenso para diversidade do uso (VCDU) para as categorias de indicação terapêutica por moradores da comunidade Manga, MA

\begin{tabular}{lc}
\hline Valor de consenso para diversidade do uso & VCDU \\
\hline Problemas no fígado & 0,36 \\
Inflamação em geral & 0,25 \\
Cicatrizante & 0,11 \\
Problemas nos rins & 0,10 \\
Gastrite & 0,06 \\
Pressão alta & 0,04 \\
Dor de cabeça & 0,04 \\
Dor de barriga & 0,04 \\
\hline
\end{tabular}

Estudos futuros são necessários para alicerçar a indicação terapêutica contra problemas hepáticos, a indicação mais citada deste estudo. Assim, aliando tanto a pesquisa etnomédica quanto a fitoquímica, pode-se elucidar essa questão. A segunda e a terceira indicações mais citadas nesta pesquisa apontam a planta como eficaz contra processos inflamatórios e como cicatrizante. As altas quantias de taninos totais encontradas por Monteiro et al. (2014) podem suportar essas indicações, já que os taninos podem conferir ações anti-inflamatórias e cicatrizantes. Muitas plantas arbóreas ou arbustivas são bem reconhecidas e usadas por tais ações e por diferentes culturas no sertão nordestino (Albuquerque et al., 1997; Araujo et al., 2008; Monteiro et al., 2011).

Há alguns estudos sobre o uso e a atividade biológica de Lafoensia sp. (Solon et al., 2000; Galdino et al., 2009; Guimarães et al., 2010; Porfírio et al. 2009; Baptistel et al., 2014; Monteiro et al., 2014). As atividades antibacteriana e antidepressiva de L. pacari A. St.-Hil. foram verificadas (Galdino et al., 2009; Porfírio et al., 2009) e, num estudo realizado com plantas úteis do cerrado, Lima et al. (2015) verificaram atividade leishmanicida significativa. O extrato aquoso das cascas do caule de L. pacari A. St.-Hil. demonstrou atividade sedativa e antinociceptiva (Matos et al., 2008). Especificamente sobre a espécie-alvo deste estudo, não foram encontrados trabalhos sobre sua atividade biológica.

Entre os informantes, a parte mais indicada como usada foram as cascas do caule, com valor de consenso para parte da planta 0,53 (56\% das indicações), seguidas das folhas, com valor de consenso 0,46 (44\% das indicações). Não houve distinções significativas $(\mathrm{H}=0,16 ; \mathrm{p}=0,68)$.

As cascas do caule de algumas espécies nativas já tiveram sua importância medicinal bem documentada na literatura pesquisada, principalmente no Nordeste (Albuquerque et al., 1997; Araujo et al., 2008; Baptistel et al., 2014; Monteiro et al., 2014). Algumas dessas plantas têm grandes quantidades de cascas do caule coletadas e comercializadas em mercados e feiras públicos do Nordeste do Brasil, onde são apontadas como curativas devido aos altos teores de compostos fenólicos do metabolismo 
secundário, especificamente os taninos. Monteiro et al. (2014) encontraram cerca de $75 \mathrm{mg} / \mathrm{g}$ de taninos totais (aproximadamente 7,3\% do peso total seco) nas cascas do caule de L. replicata, alicerçando suas indicações de uso. Na comunidade Santo Antônio, Piauí, os informantes relataram 13 usos para cascas do caule, ressaltando um alto valor de uso para a planta. Os autores afirmaram que essa espécie ficou entre os dez maiores valores de uso calculados, denotando uma alta importância relativa. Contudo, vale ressaltar a relevância do uso das folhas apontada neste trabalho. Muitas árvores são usadas como medicinais, atribuindo-se somente às cascas seu poder curativo (Zschocke et al., 2000; Monteiro et al., 2006). Essas plantas estão submetidas à coleta agressiva de cascas, podendo formar um anelamento, prática que fatalmente atinge o vegetal. Além do mais, tanto o crescimento como a reprodução dessas espécies arbóreas mais populares são lentos (Zschocke et al., 2000), e o mesmo ocorre com muitas das plantas medicinais da caatinga. Então, as folhas podem ser um importante substituto na terapia local, diminuindo, assim, a pressão agressiva de coleta em torno das cascas. Reforçando essa perspectiva, Guimarães et al. (2010) encontraram no extrato etanólico de folhas de L. pacari Pohl. promissores efeitos analgésico e anti-inflamatório em camundongos. Sendo assim, argumentaram também que o extrato etanólico das folhas são substitutos possíveis para as cascas, tão reconhecidas pela medicina popular.

\subsection{Modo de preparo, forma de}

administração e restrições ao uso da espécie L. replicata Pohl. na comunidade Manga, $M A$

O chá foi o modo de preparo mais citado, com 17 (43\%) citações no total. Tanto as cascas como as folhas são utilizadas na confecção do chá, de acordo com os informantes. Logo em seguida apareceu o molho (cascas e folhas são mergulhadas em água), com 11 (34\%) citações. Houve cinco (15\%) citações para chá ou molho, sem distinção, e duas para preparo de garrafada (6\%). Na medicina tradicional, o decocto e também a infusão de partes vegetais de algumas dessas espécies - por exemplo, Stryphnodendrum sp. e Myracrodruon urundeuva Allemão - são bem documentados (Baptistel et al., 2014; Oliveira et al., 2014). Contudo, ainda há lacunas de informaçoes referentes ao uso medicinal da espécie deste estudo.

Todos os informantes indicaram consensualmente que o remédio preparado a partir da planta deve ser administrado por via oral. Um informante, entretanto, acrescentou o uso tópico, sendo o molho utilizado para lavar os ferimentos.

Quanto às restrições ao uso de L. replicata Pohl., pouco mais da metade dos informantes, 17 especificamente $(53 \%)$, mencionou que a planta não apresenta restrições ao consumo. Contudo, dez (31\%) entrevistados afirmaram que o tratamento é contraindicado durante a gravidez e, ainda, cinco $(15 \%)$ pessoas não recomendaram o uso para crianças. No extrato etanólico das cascas do caule de L. pacari A. St.-Hil., foi detectada a presença de taninos, esteroides, triterpenos e saponinas (Solon, 2000). Sendo assim, estudos sobre a toxicidade, tanto de L. pacari A. St.-Hil. quanto de L. replicata Pohl., são necessários. A presença de compostos como saponinas pode conferir toxicidade à planta.

\section{Considerações finais}

Os resultados demostraram que os entrevistados possuem considerável conhecimento sobre as propriedades terapêuticas da espécie em questão. 
Diante disso, estudos vindouros sobre eficácia, estabelecimento de dosagens e sua toxicidade são interessantes e necessários. No caso específico dessa espécie, folhas e cascas foram indicadas sem preferências entre as partes. Isso pode ser interessante para o estabelecimento de projetos de manejo, ao direcionar o uso terapêutico às folhas, já que a coleta de cascas se dá de maneira agressiva para a planta, afetando sua integridade e até sua estrutura populacional.

Há na literatura disponível algumas pesquisas enfocando os usos e as atividades de $L$. pacari A. St.-Hil. Dessa forma, este estudo recomenda também $L$. replicata Pohl. como uma potencial espécie terapêutica.

\section{Referências}

Agra, M. F.; Baracho, G. S.; Nurit, K.; Basílio, L. J. D.; Coelho, V. P. M. Medicinal and poisonous diversity of the flora of "Cariri Paraibano", Brazil. Journal of Ethnopharmacology, 111, 383-395, 2007.

Albuquerque, U. P. Etnobotânica: uma aproximação teórica e epistemológica. Revista Brasileira de Farmácia, 78(3), 60-64, 1997.

Albuquerque, U. P.; Ramos, A. M.; Lucena, R. F. P.; Alencar, N. L. Methods and techniques used to collect ethnobiological data. In: Albuquerque, U. P.; Cunha, L. V. F. C.; Lucena, R. F. P.; Alves, R. R. N. (Orgs.). Methods and techniques in Ethnobiology and Ethnoecology. Springer, New York, 2014. p. 15-38.

Almeida, C. F. C. B. R.; Cabral, D. L. V.; Amorim, E. L. C.; Araújo, J. M.; Albuquerque, U. P. Comparative study of the antimicrobial activity of native and exotic plants from the Caatinga and Atlantic Forest selected through an ethnobotanical survey. Pharmaceutical Biology, 50, 201-207, 2012.

Araujo, T. A. S.; Alencar, N. L.; Amorim, E. L. C.; Albuquerque, U. P. A new approach to study medicinal plants
Salienta-se também a riqueza da flora local, distinta por áreas ecotonais entre caatinga e cerrado. Esse aspecto oferece rica oportunidade para o estabelecimento de pesquisas abrangendo o escopo da biodiversidade vegetal e o conhecimento tradicional associado (Farias, 2003; Baptistel et al., 2014). A região ainda necessita de pesquisas etnodirigidas com abordagens diferenciadas, tais como a influência de fatores culturais ou socioeconômicos - idade, gênero e ocupação das pessoas - na distribuição do conhecimento e no uso dessas plantas úteis, bem como de fatores ecológicos, como, por exemplo, identificação e contagens de indivíduos e quantidade de partes da planta coletada. Essas informações podem alicerçar propostas de manejo direcionadas às espécies vegetais muito demandadas localmente.

with tannins and flavonoids contents from the local knowledge. Journal of Ethnopharmacology, 120, 72-80, 2008.

Ayres, M.; Ayres Junior, M.; Ayres, D. L.; Santos, A. A. S. BioEstat 5.0: aplicações estatísticas nas áreas das ciências biológicas e médicas. Belém, PA, 2007. 364 p.

Barros, F. N.; Nascimento, V. T.; Medeiros, P. M. Ethnobotany and population status of Myracrodruon urundeuva Allemão in rural Northeastern Brazil. Economic Botany, 70, 2016. doi:10.1007/s12231-015-9329-4

Begossi, A.; Hanazaki, N.; Tamashiro, J. Y. Medicinal plants in the Atlantic Forest (Brasil): knowledge, use and conservation. Human Ecology, 30(3), 281-299, 2002.

Byg, A.; Baslev, H. Diversity and use of palms in Zahamena, Eastern Madagascar. Biodiversity and Conservation, 10, 951-970, 2001.

Christo, A. B.; Guedes-Bruni, R. R.; Fonseca-Kruel, V. S. Uso de recursos vegetais em comunidades rurais limítrofes à reserva biológica de Poço das Antas, Silva Jardim, Rio de Janeiro: estudo de caso na gleba Aldeia Velha. Rodriguésia, 57(3), 519-542, 2006. 
Farias, R. R. S. Florística e fitossociologia em trechos de vegetação do Complexo de Campo Maior/Piauí. Recife, Dissertação (Mestrado) - UFPE, 2003.

Galdino, P. M.; Nascimento, M. V. M.; Sampaio, B. L.; Ferreira, R. N.; Paula, J. R.; Costa, E. A. Antidepressant-like effect of Lafoensia pacari A. St.-Hil. ethanolic extract and fractions in mice. Journal of Ethnopharmacology, 124, 581-585, 2009.

Giraldi, M.; Hanazaki, N. Uso e conhecimento tradicional de plantas medicinais no Sertão do Ribeirão, Florianópolis/ SC, Brasil. Acta Botanica Brasilica, 24, 395-406, 2010.

Guimarães, H. A.; Nascimento, M. V. M.; Tavares, A.; Galdino, P. M.; Paula, J. R.; Costa, E. A. Effects of ethanolic extract of leaves of Lafoensia pacari A. St.-Hil., Lythraceae (pacari), in pain and inflammation models. Revista Brasileira de Framacognosia, 20(3), 328-333, 2010.

IBGE - Instituto Brasileiro de Geografia e Estatística. Disponível em: <http://www.ibge.gov.br/>. Acesso em: maio 2014.

Lima, P. C.; Santos, M. G.; Calabrese, K. S.; Silva, A. L. A.; Almeida, F. Avaliação da capacidade leishmanicida de espécies vegetais do cerrado. Revista de Patologia Tropical, 44(1), 45-55, 2015.

Lucena, C. M.; Costa, G. M.; Souza, R. F.; Carvalho, T. K. N.; Marreiros, N. A.; Alves, C. A. B.; Pereira, D. D.; Lucena, R. F. P. Conhecimento local sobre cactáceas em comunidades rurais na mesorregião do sertão da Paraíba (Nordeste, Brasil). Biotemas, 25, 281-291, 2012.

Melo, J. G.; Santos, A. G.; Nascimento, S.; Amorim, E. L. C.; Albuquerque, U. P. Medicinal plants as anti-tumor agents in Brazil: an ethnobotanical approach. Evidence-Based Complementary and Alternative Medicine, 2011a. doi: $10.1155 / 2011 / 365359$

Melo, J. G.; Santos, A. G.; Nascimento, S.; Amorim, E. L. C.; Albuquerque, U. P. Phytochemical and pharmacological notes of plants indicated to treat tumors in Brazil. Revista Brasileira de Farmacognosia, 21, 744-753, 2011 b.

Monteiro, J. M.; Albuquerque, U. P.; Lins-Neto, E. M. F.; Araújo, E. L.; Amorim, E. L. C. Use patterns and knowledge of medicinal species among two rural communities in Brazil' semi-arid northeastern region. Journal of Ethnopharmacology, 105, 173-186, 2006.
Monteiro, J. M.; Ramos, M. A.; Araújo, E. L.; Amorim, E. L. C.; Albuquerque, U. P. Dynamics of medicinal plants knowledge and commerce in an urban ecosystem (Pernambuco, Northeast Brazil). Environmental Monitoring and Assessment, 178, 179-202, 2011.

Monteiro, J. M.; Souza, J. S. N.; Lins Neto, E. M. F.; Scopel, K.; Trindade, E. F. Does total tannin content explain the use value of spontaneous medicinal plants from the Brazilian semi-arid region? Revista Brasileira de Farmacognosia, 24, 116-123, 2014.

Oliveira, D. R.; Ferreira Júnior, W. S.; Nilo Bitu, V. S.; Pinheiro, P. G.; Menezes, C. D. A.; Brito Junior, F. E.; Albuquerque, U. P.; Kerntopf, M. R.; Coutinho, H. D. M., Fachinetto, R.; Menezes, I. R. A. Ethnopharmacological study of Stryphnodendron rotundifolium in two communities in the semi-arid region of northeastern Brazil. Revista Brasileira de Farmacognosia, 24, 124-132, 2014.

Porfírio, Z.; Melo Filho, G. C.; Alvino, V.; Lima, M. R. F.; Sant'Ana, A. E. G. Atividade antimicrobiana de extratos hidroalcoólicos de Lafoensia pacari A. St.-Hil., Lythraceae, frente a bactérias multirresistentes de origem hospitalar. Revista Brasileira de Farmacognosia, 19(3), 785-789, 2009.

Ramos, M. A.; Albuquerque, U. P. The domestic use of firewood in rural communities of the Caatinga: how seasonality interferes with patterns of firewood collection. Biomass \& Bioenergy, 39, 147-158, 2012.

Silva, F. S.; Ramos, M. A.; Hanazaki, N.; Albuquerque, U. P. Dynamics of traditional knowledge of medicinal plants in a rural community in the Brazilian semi-arid region. Revista Brasileira de Farmacognosia, 21(3), 382-391, 2011.

Soldati, G. T.; Albuquerque, U. P. A new application for the optimal foraging theory: the extraction of medicinal plants. Evidence-Based Complementary and Alternative Medicine, 2012, 1-10, 2012. doi: 10.1155/2012/364564

Solon, S.; Lopes, L.; Sousa Jr., P. T.; Schmeda-Hirschmann, G. Free radical scavenging activity of Lafoensia pacari. Journal of Ethnopharmacology, 72, 173-178, 2000.

Titiev, M. Introdução à antropologia cultural. Lisboa: Fundação Calouste Gulbenkian, 2000.

Torres-Avilez, W. M.; Nascimento, A. L. B.; Campos, L. Z. O.; Silva, F. S.; Albuquerque, U. P. Gênero e idade. In: Albuquerque, U. P. (Org.). Introdução à etnobiologia. Recife: NUPEEA, 2014.189 p. 
Voeks, R. A. Are women reservoirs of traditional plant knowledge? Gender, ethnobotany and globalization in Northeast Brazil. Singapore Journal of Tropical Geography, 28, 7-20, 2007.

Voeks, R. A.; Leony, A. Forgetting the forest: assessing medicinal plant erosion in eastern Brazil. Economic Botany, 58, S294-S306, 2004.

Voeks, R. A.; Nyawa, S. Healing flora of the Brunei Dusun. Borneo Research Bulletin, 32, 178-195, 2001.
WHO - World Health Organization. International Statistical Classification of Diseases and Related Health Problems. $10^{\text {th }}$ revision. Disponível em: $<\mathrm{http}: / /$ www.who.int/classifications/apps/icd/icd10online/>. Acesso em: dez. 2014.

Zschocke, S.; Rabe, T.; Staden, J. Plant part substitution - a way to conserve endangered medicinal plants? Journal of Ethnopharmacology, 71, 281-292, 2000. 